\title{
CLINICAL VERSUS RADIOGRAPHIC SUCCESS OF NECROTIC PRIMARY MOLARS TREATED BY LESION STERILIZATION AND TISSUE REPAIR. A RANDOMIZED CONTROLLED TRIAL
}

\author{
Mahmoud Magdy Mohamed Saadoon*, Mohamed Medhat Kataia* \\ Amr ezzat abdellaatif** and Samah Awad**
}

\begin{abstract}
The success and failure after treatment of necrotic primary molars causes a hassle to clinicians. This study aims to study the difference between clinical success and radiographic success after treatment using Lesion Sterilization and Tissue Repair technique. The study was conducted on 41 subjects in each group, all subjects were treated by LSTR technique. At the end of the study despite of radiographic failure in $21 \%$ from group A \& $4 \%$ from group B. Molars were clinically successful with absence of pain, swelling, mobility, fistula formation, and were maintained functioning till the end of the study.
\end{abstract}

\section{INTRODUCTION}

Primary molars with necrotic pulp or abscess are common unfortunate event faced in pediatric dentistry. The traditional treatment is either pulpectomy or extraction (McDonald et al., 2004). According to the American Academy of Pediatric Dentistry (AAPD) 2008, success of pulpectomy treatment is about $85 \%$ in abscessed primary teeth.

A newly introduced treatment options is using a mixture of antibiotics for disinfection of root canal. LSTR is a non-instrumentation endodontics employs removal of coronal pulp only and using mixture of antibiotics for disinfection of pulpal and periapical lesion. In this study we used simvastatin which is a well-known anti-hyperlipdemia drug together with triple mix antibiotics composed of metronidazole, cefixime, ciprofloxacin. Aminabadi et al., 2015 proposed the term 3mix-tatin as an acronym for the mixture of triple mixture antibiotics and simvastatin. Statin components are emerging material in regenerative endodontics. Clinical and experimental trials proved the pleiotropic effect of statin, as it increases the osteoblastic function and suppresses the osteoclastic function. In addition to that, evidence proved that it is a potent anti -inflammatory as it reduce the CRP and proinflammatory cytokines (Mundy et al., 1999; Aminabadi et al ., 2015).

\footnotetext{
* Associate Professor and Head of Endodontic Department, British University. Egypt.

** Professor of Pedodontics, Cairo University, Egypt
} 


\section{MATERIALS AND METHODS}

\section{Aim of study}

The aim of the current study is to compare the clinical and radiographic success versus clinical success in necrotic primary molars treated by using lesion sterilization and tissue repair LSTR technique

\section{Sample size:}

The aim of the current study was to compare clinical and radiographic success after management of necrotic primary molars treated by lesion sterilization and tissue repair LSTR technique. We needed to study 41 case patients and 41 control patients to be able to reject the null hypothesis that the exposure rates for case and controls are equal with probability (power) 0.8 . The Type I error probability associated with this test of this null hypothesis is 0.05 . We used an uncorrected chisquare statistic to evaluate this null hypothesis. This number should be increased to total number (90) $10 \%$ more than calculated to compensate for losses during follow up.

\section{Recruitment strategy:}

- Patient was selected from the outpatient clinic of pediatric Dentistry and Dental Public Health Department- Cairo University.

\section{Allocation Implementation, Randomization and concealment:}

The allocation sequence by random allocation software version 1.0,May 2004 was generated on operator personal computer.select the number groups (2) then enter the name of each group (3-MIX-TATIN \&METAPEX). Enter the sample size (82) and select Generate from the Sequence menu. Each participant was randomly assigned following simple randomization procedure by using shuffled closed white envelops(82), which assign the number of the groups to be followed. Those closed envelops include (82) paper charts, which are folded eight times not to show its contents to assure allocation concealment. It was withdrawn by parents or patient.

\section{Lesion Sterilization and Tissue Repair (LSTR) USING 3-MIX antibiotic paste}

- 3Mix was prepared by mixing threecommercially available antibiotics with simvastatin. After removing the coating materials, 3 types of antibiotics are pulverized by porcelain mortars and pestles to achieve fine powders. A total of $100 \mathrm{mg}$ ciprofloxacin, $100 \mathrm{mg}$ metronidazole, and $100 \mathrm{mg}$ cefixime will be mixed in a ratio of 1: 1: 1. Preparation of 3Mix was trained and supervised by an expert pharmacist. 3Mixtatin was stored in a tightly capped porcelain container, adding a small amount of silica gel in a bag inside the container to maintain low humidity. This powder was mixed with normal saline to form a creamy paste of 3 Mix at the time of application (Aminabadi et al., 2015).

- Local anesthesia using mepivicaine hydrochloride $3 \%$ (septodont, saint-maurdes Fosses, France) was administered.

- Rubber dam isolation.

- Access cavity was prepared with a fissure bur in a high-speed handpiece, necrotic pulp tissue was removed using a sterile sharp spoon excavator.

- The canal orifices was enlarged with a round bur (one $\mathrm{mm}$ diameter and two $\mathrm{mm}$ depth) to create medication receptacles.

- Cotton pellet moistened with $2.5 \%$ sodium hypochlorite $(\mathrm{NaOCl})$ was placed in the pulp chamber for 1 minute.

- Bleeding, if present, was controlled by applying sterile cotton pellet moistened with $10 \%$ sodium hypochlorite against the pulp stumps and was maintained for $1 \mathrm{~min}$.

- The 3-Mix-Tatin was mixed with saline to obtain a creamy mix and placed over the canal orifices and the pulpal floor. 

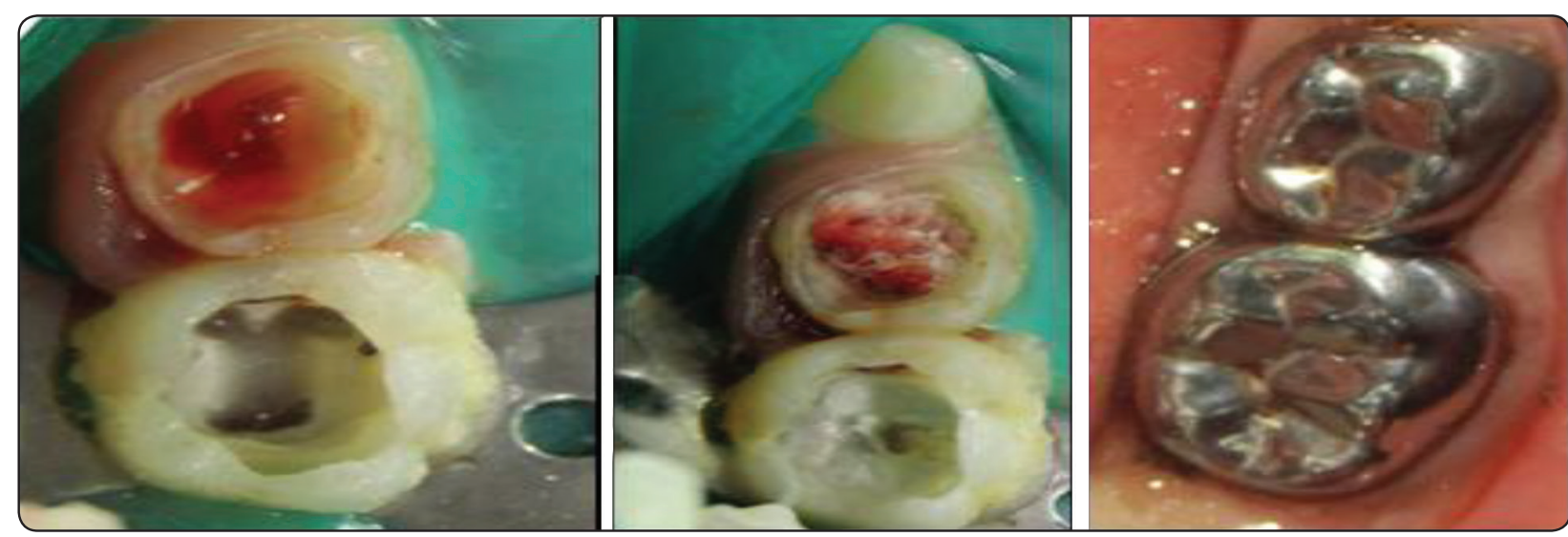

Fig. (1) Access cavity and medication cavity preparation, 3-mixtatin in medication receptacles, stainless steel crowns restoration.

- The access opening was sealed with a glass ionomer cement (Fugi IX, GC America, Alsip, Ill., USA), and the tooth was restored with a stainless steel crown [(SSC); 3M ESPE Unitek Stainless Steel Crowns, St. Paul,Minn., USA].

\section{RESULTS}

The present study evaluated the clinical and radiographic outcomes of two different materials in management of one hundred necrotic primary molars in healthy children aged from 4-8 years old (mean age $6.08 \& 5.79$ years for group A and group $\mathrm{B}$ respectively). Children were selected randomly from the outpatient clinic of Pediatric Dentistry Department, Faculty of Dentistry-Cairo University. All cases were evaluated according to clinical and radiographic criteria at 3, 6, 9 months.

The children were divided into 2 groups; Group A: using calcium hydroxide iodoform paste (Metapex), and group B using 3-Mixtatin.

TABLE (1) Number of cases at different follow up periods

\begin{tabular}{|l|l|l|l|l|}
\hline & First visit & $3^{\text {rd }}$ month & $6^{\text {th }}$ month & $9^{\text {th }}$ month \\
\hline Group A & 50 & 44 & 44 & 42 \\
\hline Group B & 50 & 44 & 44 & 42 \\
\hline
\end{tabular}

Clinical failure was considered when patients reported outcomes like pain, facial cellulitis, or mobility grade III. As a result the treatment was shifted to either extraction and space maintainer or pulpectomy. Throughout the follow up period $8(17.3 \%)$ subjects from group (A) were reported as clinical failures, compared to $10(20 \%)$ subjects in group (B).

Time of failure was different in both groups, failure at the first month following treatment was higher in 3-Mixtatin group as 4 cases were reported with flare up of infection, and presented with facial cellulitis \& pain with or without mobility. Treatment was shifted immediately to partial pulpectomy using Metapex. In Metapex group, only one case was reported with flare up of infection in the first month. At 9th month follow up 2 cases in group (A) showed mobility grade III and were extracted \& 2 cases in group (B) were reported clinical failure and was treated by pulpectomy.

TABLE (2) Time of clinical failure.

\begin{tabular}{|l|c|c|}
\hline Time & Group A & Group B \\
\hline First month & 1 & 4 \\
\hline $3^{\text {rd }}$ month & 1 & 0 \\
\hline $6^{\text {th }}$ month & 2 & 2 \\
\hline $9^{\text {th }}$ month & 4 & 4 \\
\hline
\end{tabular}


TABLE (3): Radiographic results of group A \& B (perprotocol results).

\begin{tabular}{|c|c|c|c|c|c|c|c|c|}
\hline & \multicolumn{2}{|c|}{ Furcation } & \multicolumn{2}{|c|}{ Int/Ex root resorption } & \multicolumn{2}{|c|}{ Periapical } & \multicolumn{2}{|c|}{ Pathology } \\
\hline & Group A & Group B & Group A & Group B & Group A & Group B & Group A & Group B \\
\hline \multirow{3}{*}{ 3rd month } & $24 / 44$ & $26 / 44$ & $10 / 44$ & $0 / 44$ & $6 / 44$ & $4 / 44$ & $2 / 44$ & $0 / 44$ \\
\hline & $(54.5 \%)$ & $(59.1 \%)$ & $(22.7 \%)$ & $(0.0 \%)$ & $(13.6 \%)$ & $(9.1 \%)$ & $(4.5 \%)$ & $(0.0 \%)$ \\
\hline & \multicolumn{2}{|c|}{0.667} & \multicolumn{2}{|c|}{0.001} & \multicolumn{2}{|c|}{0.739} & \multicolumn{2}{|c|}{0.494} \\
\hline \multirow{3}{*}{ 6th month } & $18 / 44$ & $12 / 44$ & $18 / 44$ & $6 / 44$ & $6 / 44$ & $4 / 44$ & $2 / 44$ & $0 / 44$ \\
\hline & $(40.9 \%)$ & $(27.3 \%)$ & $(40.9 \%)$ & $(13.6 \%)$ & $(13.6 \%)$ & $(9.1 \%)$ & $(4.5 \%)$ & $(0.0 \%)$ \\
\hline & \multicolumn{2}{|c|}{0.177} & \multicolumn{2}{|c|}{0.004} & \multicolumn{2}{|c|}{0.739} & \multicolumn{2}{|c|}{0.494} \\
\hline \multirow{3}{*}{ 9th month } & $18 / 42$ & $14 / 42$ & $18 / 42$ & $6 / 42$ & $6 / 42$ & $8 / 42$ & $0 / 42$ & $0 / 42$ \\
\hline & $(42.9 \%)$ & $(33.3 \%)$ & $(42.9 \%)$ & $(19.3 \%)$ & $(14.3 \%)$ & $(19 \%)$ & $(0.0 \%)$ & $(0.0 \%)$ \\
\hline & \multicolumn{2}{|c|}{0.369} & \multicolumn{2}{|c|}{0.004} & & & \multicolumn{2}{|c|}{---- } \\
\hline
\end{tabular}

i.e.n/total number of group (percentage)

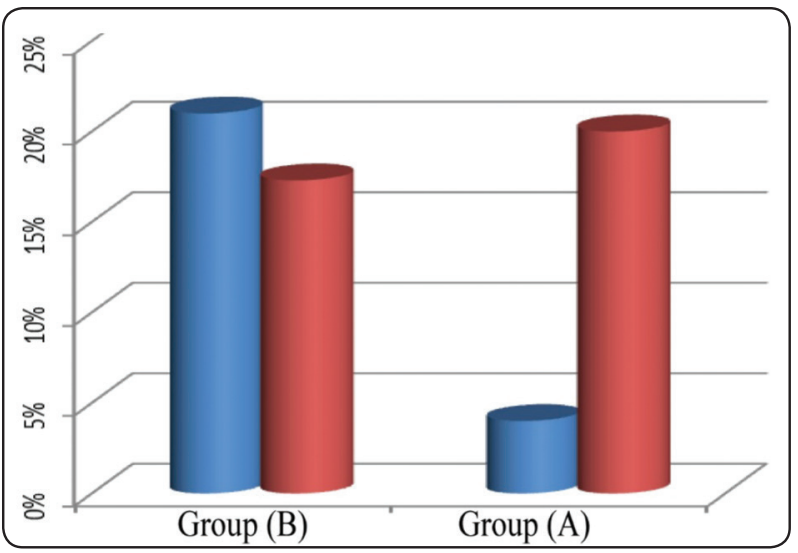

Fig. (2) Clinical failure, and radiographic failure despite of clinical success between the study groups (per protocol).

Clinical success rate at 9th month follow up for Metapex group (A) was $82.7 \%$, and for 3- Mixtatin group (B) was $80 \%$, ARR was -0.02 which means that success rate in group (A) was $2 \%$ higher than in group (B).

Radiographic failure was considered when there was furcation radiolucency, periapical radiolucency, periapical pathology, or increasing resorptive process. Despite of clinical success radiographic failure was found in $10(21 \%)$ subjects from group
(A) compared to 2(4\%) subjects from group (B).

Bone regeneration at previously resorbed furaction area was evident by reduction in number of subjects with furcation radiolucency from time of recruitment to time of last follow up after 9 months. In group (A) $33 \%$ of subjects showed healing or bone regeneration at furcation radiolucency area, compared to $47.3 \%$ in group (B)

\section{DISCUSSION}

The target of pediatric dentistry in management of primary dentition is to maintain the integrity of dental arch as possible till the time of shedding for promoting function, esthetic, phonetics and avoidance possible space problems (McDonald et al., 2011b). Endodontic management of primary teeth with severe pulpal necrosis is evidenced by non-vital pulp tissue, fistula, and loss of alveolar bone is generally challenging. Though several reasons have been cited for this, the main one being morphology of the primary teeth which is not suitable for biomechanical preparation (Saltzman 2005). 
In this study clinical failure was considered when subjects were reported with outcomes like pain, facial cellulitis, or mobility grade III. Clinical failure was reported in $8(17.3 \%)$ subjects from group (A), compared to $10(20 \%)$ subjects from group (B). Unlike Prabhakar, 2008 study which showed 3.3\% \& Aminabadi et al., 2016 which showed $3.2 \%$. In both studies the sample size was smaller. In the former study a different mixture of antibiotics was used and in the latter study radicular pulp was removed and presence of necrotic pulp wasn't an inclusion criteria. Unlike this study where coronal pulp only was removed, also inclusion criteria included necrotic pulp \& preoperative pain.

\section{CONCLUSIONS}

From the results of this study, the following conclusions were obtained:

1. The 3 mix could be a successful alternative to Metapex in the light of overall success and radiographic results.

2. Despite radiographic failure in $21 \%$ from group A \& 4\% from group B, molars were clinically successful with absence of pain, swelling, mobility, fistula formation, and were maintained functioning till the end of the study.

\section{REFERENCE}

1. Aminabadi Asl, N., Maljaei, E., Erfanparast, L., Ala Aghbali, A., Hamishehkar, H., \& Najafpour, E. (2013). Simvastatin versus Calcium Hydroxide Direct Pulp Capping of Human Primary Molars: A Randomized Clinical Trial. Journal of Dental Research, Dental Clinics, Dental Prospects, 7(1), 8-14. http://doi.org/10.5681/joddd. 2013.002

2. Mundy G, Garrett R, Harris S, et al(1999). Stimulation of bone formation in vitro and in rodents by statins. Science.; 286:1946-1949. doi: 10.1126/science. 286. 5446. 1946.

3. McDonald RE, Avery DR, Dean JA.(2011b) Treatment of deep caries, vital pulp exposure, and pulpless teeth. In: McDonald RE, Avery DR, eds. Dentistry for the Child and Adolescent. 9th ed. Philadelphia, Pa: CV Mosby Co; 2004:388-412.

4. Prabhakar AR, Sridevi E, Raju OS, Satish V(2008). Endodontic treatment of primary teeth using combination of antibacterial drugs: An in vivo study. indian Soc Pedod Prev Dent;26(Suppl 1): 5-S10. 